\title{
Bioavailability, Bioequivalence, and Pharmacokinetics: Clinical Effectiveness in Drug Development
}

\section{Ghazi M E Hussein ${ }^{1}$, Heyam Saad Ali $^{2 *}$ and Babiker M Elhaj ${ }^{3}$}

${ }^{1} R \& D$ - Pharmacology, JAPAN Division of Pharmacology, Department of

Pharmacognosy and Natural Medicines, Kyoto Pharmaceutical University, Japan

${ }^{2}$ Department of Pharmaceutics, College of Pharmacy, University of Khartoum,

Khartoum, Sudan

${ }^{3}$ Department of Pharmaceutical Sciences, College of Pharmacy and Health Sciences,

University of Science and Technology of Fujairah, Fujairah, UAE

*Corresponding Author: Heyam Saad Ali, Department of Pharmaceutics, College of Pharmacy, University of Khartoum, Khartoum, Sudan.
Received: August 09, 2021

Published: September 25, 2021

(C) All rights are reserved by Heyam Saad Ali., et al.

\begin{abstract}
Bioequivalence has also been referred to as comparative bioavailability. The concept of bioequivalence started gaining an increased attention during the last three decades, after it became evident that some marketed products containing the same amount of drug and marketed in the same dosage form exhibited marked differences between their therapeutic responses. In many instances, different therapeutic responses observed with these products were correlated successfully to dissimilar levels of drug concentration in the plasma, which was caused mainly due to differences in the rate of absorption of drug from these products. It is now very well established that the rate and extent to which an administered drug dose, to cause a systemic effect, must reach systemic circulation first, therefore its bioavailability depends on a number of very important factors. Although, the previous revisions specifies comparison of pharmaceutical equivalents. It is obvious that pharmaceutical alternatives are also included because the comparison here is also between therapeutic drug ingredients as well.

Conclusion: Bioavailability testing for all products is economically difficult, and for some drugs such studies may not necessarily be essential. The question of determining bioavailability, however, is of particular interest for drug products which fall into distinct categories.
\end{abstract}

Keywords: Bioequivalence; Bioavailability; Clinical Effectiveness

\section{Background}

From a pharmaceutical standpoint, there are three inherent factors that are known to affect absorption for systemic availability of a drug following administration of the drug dose [1].
These are:

- Formulation techniques used in the development of the dosage form.

- Methods and equipment used in the manufacture of the dosage form 
- Materials used in the formulation of the dosage form. Despite being inert, they are associated to various issues that are suspected to cause bioavailability problems, such as: the particle size and crystal form or polymorphic form of the drug substance, diluents and excipients used in the dosage form, including, binders, disintegrating agents, fillers, lubricants, coating materials solvents, suspending agents and dyes, Lubricants and coating materials, are among the materials [2].

According to FDA definition, bioequivalent drug products are those pharmaceutical equivalents that display comparable rate and extent of absorption of therapeutic drug moiety when studied under similar experimental conditions. FDA required conditions include (but are not limited to) the following:

- AUC should not show significant difference from the reference drug, with the same molar concentration and conditions of the experiment, whether the dose is single or multiple. The similarity in the rate and extent of absorption is established when the difference in the rate of absorption from the reference drug is intentional and is reflected in the proposed labeling of the test drug [3].

- This is acceptable only if difference in the rate of absorption is not essential to the attainment of effective concentration of drug in the body (chronic cases that need special consideration) [4].

- For topically applied products intended for local rather than systemic effect, other in vivo tests of bioequivalence may be appropriate.

- Bioequivalence may be assessed by in-vitro standards, using a suitable in vitro bioequivalence standard, when correlated to human in-vitro tests data [5].

The formulation of the same drug by different manufacturers in similar dosage forms, or the formulation of a drug by the same manufacturer in different countries may exhibit different bioavailability characteristics and therefore potentially different clinical effectiveness. For example, two products may appear "identical or 'equivalent', because they contain the same drug, in same dosage strength, and in the same dosage form. But, if the formulation materials (e.g. diluents, binders. disintegrants, lubricants, etc.) used in their manufacture were different, then it is possible that these products may exhibit wide differences in bioavailability of the drug and thus in clinical effectiveness of the drug [6].

The classic case of Dilantin is an example of this situation, A few years ago, a number of Dilantin toxicity cases were reported in Australia, The patients who reported bad toxicity, had been taking Dilantin for some time not only in the same dosage form, but also in the same strength of the dosage form.

Later, it was found that the company had started manufacturing Dilantin locally, but because of availability problems, a different diluent was used in the domestic product, The change in diluent was identified to be responsible for the different bioavailability of drug from the locally manufactured dosage form because the diluent previously used was binding a fraction of the drug in the dosage Form. Thus, due to no binding of drug to the new diluent, a larger amount of drug was released from the new formulation [7].

In another case, digoxin was shown to exhibit varying bioavailability from different manufacturers although each dosage form contained the same drug in the same strength.

Also, differences in the bioavailability of digoxin from different batches of the same dosage form made by the same manufacturer were reported in several studies published in scientific journals. Many researches have shown that a variety of factors in the preparation of formulation can result in differing bioavailability of the drug product and possibly clinical effectiveness. These factors include: use of different formulation materials, use of similar material obtained from different suppliers, validity of process variable, method of manufacture and equipment used in the manufacturing process. Thus, equivalency of drug products which may appear identical in many respects becomes questionable when drugs are marketed by more than one pharmaceutical manufacturer. The study of biopharmaceutics gives substantial evidence that the manufacturing method gives information of the bioavailability of the final drug product.

Equivalence

Equivalence is a comparative term and is used to indicate that two or more products labeled as equivalent are presumed to be similar to one another with respect to a specific characteristic or function. The term bioequivalence is used in pharmaceutical industry 
to indicate similarities in different products or dosage forms with respect to defined set standards. The following terms are used to define the type or level of "equivalency" between drug products [8].

\section{Bioequivalence}

Many drugs are marketed by more than one pharmaceutical manufacturer, but all are in compliance with FDA standards of the approved bioavailability, an equivalent therapeutic effect to the brand product, despite variation in the used additives and method of manufacturing [9].

Other terms are associated with bioavailability and bioequivalence include: pharmaceutical equivalents, pharmaceutical alternatives, bio-logical equivalents, and therapeutic equivalents to explain the level of equivalency, they do not necessarily imply that all bioequivalent products must contain the same inert ingredients [10].

Despite this, however, in most cases pharmaceutical equivalent products are also bioequivalent as well as therapeutically equivalent. The differences arise only when the active ingredient is "incompatible" with one or more of the inert ingredients. For example, a tetracycline dosage form prepared using no calcium containing ingredient will be pharmaceutically equivalent to calcium-containing ingredients but in all probability will be neither bioequivalent nor therapeutically equivalent [11].

Until the late 1960's, disintegration time of tablets was considered an adequate test to ensure the availability of drug from the dosage form. However, in the case of some drugs, serious deficiency in bioavailability was noted to exist even if the tablet disintegrated fully in vivo. The following discussion highlights examples of wide variation in the bioavailability of some critical drugs and demonstrates serious consequences on the safety and therapeutic efficacy of some important drugs [12].

Aspirin

In a study dealing with three aspirin tablet products, it was reported that absorption rates, as well as the incidence and severity of localized gastric irritation (and possibly bleeding) following administration of aspirin tablets were interrelated [13]. Both were a function of dissolution rate of aspirin in its particular dosage form. The rank order of the in vivo results showed excellent correlation to in vitro dissolution rates of the three aspirin products [14]. The authors pointed out that disintegration time (which was the major test for in vitro bioequivalence at that time) had no bearing on the rate of absorption of aspirin tablets studied. A comparison of disintegration times and dissolution rates (or initial absorption rates) of the three products tested indicated that the product with a longer disintegration time was actually most rapidly absorbed. It can be concluded that while delayed disintegration can interfere with absorption, it does so only by its effect on dissolution. Interestingly, the rank order of absorption of the three brands of aspirin tablets (and hence the side-effects) reported coincided with an earlier study which determined gastrointestinal blood loss in 120 subjects taking various forms of salicylates. In other words, the longer the aspirin tablet stayed in the stomach, the lower the absorption, the greater the irritation and the more chance that severe side effects such as gastric bleeding can take place.

Another study investigated bioavailability of the following four dosage forms of aspirin: a solution, plain tablet, plain tablet containing alkaline additive as a buffer to enhance dissolution, and microencapsulated aspirin particles [15].

The study found that absorption from the four dosage forms occurred at widely different rates. The shortest absorption half-life of aspirin (time required for fifty percent of the dose to be absorbed) was 5 minutes and was observed when aspirin was administered as a solution dosage form. The longest absorption half-life of aspirin was 80 minutes and was found with microencapsulated aspirin particles.

Soluble formulations of aspirin are presently offered in the marketplace, the effervescent type of dosage form were required for improved solubility of aspirin, that was enabled by combination of sodium bicarbonate which resulted in faster relief of pain than plain aspirin [16].

Digoxin

Digoxin is one of the common cardiac glycosides. It shows a steep dose/response curve, which indicates a narrow window between the drug levels: the therapeutic and toxic. This becomes a critical issue from a clinical stand-point., because a narrow margin between therapeutic and toxic Levels can cause significant fluctuations in blood levels, which can result either in subclinical response or in toxic side effects [17]. 
For digoxin, the problem is exacerbated by its poor aqueous solubility and incomplete absorption from the gastrointestinal tract. Some reports indicating frequent occurrence of serious intoxication of patients under treatment with digitalis glycosides were published in the literature in the early 70's. Since digoxin is a life- saving drug, bioequivalence of digoxin tablets became a major concern $[18,19]$.

An awareness of bioequivalence of digoxin preparations was prompted by a report published in the early). It has been reported that substantial differences in dosage regimens were required to stabilize patients on digoxin tablets [20].

Subsequent bioavailability studies showed that there were four to seven fold differences in serum levels of the glycoside in the same patient not only between products of different manufacturers, but also between lots of the same manufacturer. The existence of serious bioequivalence problems with digoxin preparations was confirmed by other authors as well.

Marked differences in bioavailability of digoxin are attributed largely to changes in formulation of the product and/or its manufacturing process [21].

The fact that these changes can lead to significant modifications in dissolution rate of the tablet, and hence in bioavailability of the drug was high and had been highlighted by several reports that were published in Great Britain [22,23].

These reports indicated that a change in the manufacturing process of Lanoxin, the most widely used brand of digoxin in Great Britain, had resulted in the production and distribution of Lanoxin tablets that possessed almost double the effective potency of the batches of Lanoxin tablets that were marketed before the change [24]. Another study reported that the particle size of digoxin used in the manufacture of digoxin tablets played an important role in the bioavailability. According to this report, reduction of particle size of digoxin from $102 \mathrm{pm}$ to about $10 \mu \mathrm{g}$ resulted in doubling its bioavailability. Intensive care of serum drug concentration of digoxin my ease production with its administration problems concerning bioequivalence [25].

\section{Nitrofurantoin}

The particle size of nitrofurantoin used in the formulation of the tablet dosage form has been shown to have a very large influence on bioavailability of the drug.

Many reports pointing to bioequivalence of several nitrofurantoin products have appeared in the literature [26].

It appears that the bioequivalence problem of nitrofurantoin may have been augmented by the early USP dissolution time specification for the tablets [27].

Since rapid dissolution and absorption of nitrofurantoin can cause gastrointestinal upset and nausea, the early USP dissolution time specification of nitrofurantoin was designed to minimize these side effects. In order to prevent acceptance of drug products with extremely low bioavailability, USP XX modified the dissolution limit from “60\% must dissolve in I hr [28].

The wet crushing that condensed the particles and created microcrystalline form played a main role in speeding up of dissolution time of nitrofurantoin and so boosted its bioavailability, particularly of poor aqueous solubility [29,30].

\section{Phenytoin}

Phenytoin is an extensively used antiepileptic drug. The therapeutic serum level of phenytoin should be maintained within a narrow range $(10 \mathrm{mg} / \mathrm{L}-20 \mathrm{mg} / \mathrm{L})$ in order to assure efficacy while avoiding intoxication [31].

In 1968 several toxic episodes attributed to over dosage were reported in Australia. The manufacturer confirmed that a change had been made in one of the excipients used in the manufacture of phenytoin tablets [32].

It was discovered that the change in the excipient caused a significant increase in bioavailability of the product which led to the overdose side effect (intoxication). In the 1970's, many investigators reported wide variations in plasma levels of phenytoin marketed products). It was also reported that phenytoin preparations containing the sodium salt of the drug exhibited greater bioavailability than phenytoin products containing phenytoin as the free acid [33].

In a study dealing with the evaluation of relative bioavailability of eleven batches of phenytoin sodium capsules representing eight different manufacturers, the mean peak concentration of phenytoin in plasma of one product was found to be consistently lower 
than other products at each sampling time. Also, certain products exhibited significantly higher area under the plasma concentration versus time curve, and concentration of drug in plasma at certain sampling points than the reference [34].

Based upon various published reports citing serious bioavailability- bioequivalence problems, and because of physicochemical properties of phenytoin, its narrow therapeutic range and dose-dependent kinetics, phenytoin has been identified as a critical drug which exhibits potential bioavailability-bioequivalence problems. Therefore, because of wide divergence in dissolution rate of its marketed products and because of the slow dissolution characteristics of the most commonly prescribed reference product, the FDA and USP were prompted to designate phenytoin capsules as either extended-release or prompt-release product (USP XX, p.621, 1980) [35].

The monographs included in USP 23 - NF 18 on phenytoin are: phenytoin tablets (chewable), extended phenytoin sodium capsules, prompt phenytoin sodium capsules, and phenytoin sodium injection. Shifts between multiple generic antiepileptic drugs in certain patients may be challenging as there are variation between clinical studies about antiepileptic drugs where there should not be a problem with generic substitution [36].

\section{Prednisone}

The discovery of bioequivalence problems associated with prednisone tablets has an interesting historical background. In 1963, prednisone tablets were used successfully to control a familiar Mediterranean fever in a patient [37]. The patient was responding favorably to the treatment, but for some reason, the brand was switched during the treatment. Immediately after switching the brand, the new tablets failed to produce any relief, but when the patient was put back on the original brand, full clinical response was obtained. Upon dissolution testing, it was found that the dissolution of the biologically inactive prednisone tablets was significantly slower than that of the original brand. In the following year, a similar case was also reported with an arthritic patient [38].

Several other bioavailability and dissolution studies on commercially available prednisone tab- lets from different manufacturers showed that prednisone tablets were prone to bioavailability problems. The tablets differed in the rate of appearance of predni- sone in plasma (absorption rate), but not in the amount of prednisone that reached general circulation (extent of absorption) [39].

Although in some cases clinical effectiveness of such tablets was correlated positively to the in vitro dissolution rate, in other cases, wide differences in dissolution did not result in significant differences in the bioavailability of prednisone Studies demonstrated on evaluation of prednisolone efficacy implant for uveitis treatment longer term studies are needed to establish therapeutic potential of such delivery system for treatment of ocular inflammation $[40,41]$.

\section{Oxytetracycline}

Serious bioequivalence problems were reported for oxytetracycline in the late 60 's. A bioavailability study conducted on products from 16 lots of the antibiotic manufactured by 13 different manufacturers revealed that Terramycin, the innovator's product, had superior average serum levels than all other generic products, in spite of the fact that all lots had received approval from the FDA for marketing the product [42]. Concerned about these results, the FDA commissioned another scientific group to investigate bioavailability of all oxytetracycline products marketed in the USA [43].

This study simply confirmed that there was a serious bioequivalence problem with the marketed products of this antibiotic. Consequently, several lots of the antibiotic oxytetracycline capsules were recalled as a result of these reports. In another study about bioavailability of oxytetracycline, a dose of $5 \mathrm{mg} / \mathrm{kg}$ body weight was satisfactory for reducing enteropathy, but a dose of $10 \mathrm{mg} / \mathrm{kg}$ was needed to obtain the maximum decrease in GIT infections in pigs older than six weeks of age [44].

\section{Tetracycline}

Parallel to the episode of oxytetracycline products, serious bioequivalence problems were re- ported with the products of another important antibiotic, tetracycline [45]. A comparative bioavailability study of 12 batches of tetracycline representing 9 different manufacturers reported that the average drug serum peak levels of certain products were only one-half the concentration of others [46]. The bioequivalence problem with tetracycline was confirmed from the data of another separate study which compared blood levels of tetracycline after oral ingestion of three generic tetracycline capsules [47]. This study found that the concentration of tetracyc- 
line in plasma was significantly lower from the generic products than that obtained from the innovators' product. Also some studies investigated Ingestion of polluted milk by tetracycline residues in milk can increase fighting of microorganisms to antibiotics [48].

\section{Thyroid}

Reports of clinical ineffectiveness of a USP thyroid product were confirmed in 1962. It was reported that a generic product containing thyroid USP was clinically ineffective in treating hypothyroidism. Substitution of generic brand by levothyroxine USP in the same patients suffering from hypothyroidism produced satisfactory clinical efficacy [49].

These findings were later confirmed when bioavailability of two prominent brands of the drug in 34 patients was evaluated and the authors recommended that patients receiving thyroid preparations for the treatment of hypothyroidism should be treated consistently with a single brand, because adjustment of dose may be necessary if brands of the drug are switched [50].

Changing of a given drug by an alternative generic drug has become a public practice, especially in case of narrow therapeutic index medications; this behavior puts the effectiveness at great risk [51].

\section{Tolbutamide}

The clinical failure of some generic tolbutamide tablets was reported in the early), and the bioequivalence problem with tolbutamide tablets was confirmed by other studies in the following year. Later, one study reported that the time for in vitro dissolution of $50 \%$ of the drug for 26 batches of tolbutamide tablets representing 21 manufactures varied from as little as 3 minutes to as much as 120 The implication of such wide range in tolbutamide dissolution on the clinical effectiveness of the drug upon switching from one brand to another was evident Even a minor change in the formulation can have a significant effect on the bioavailability and clinical efficacy of the drug product [52].

A well-controlled clinical study compared the brand name product, Orinase, and an experimental tablet formulation containing half the amount of disentegrant [Vee Gum]. In study ten healthy subjects who don't suffer diabetes subjects had been enrolled. Bioavailability was measured by drug serum levels, and therapeutic efficacy was measured by the ability to lower serum glucose levels.
The area under curve over an 8-hr period was 3.5 times greater with Orinase than with the experimental tablet, and the difference between average glucose level for the two formulations differed markedly at the $1.5 \mathrm{hr}$ and $3 \mathrm{hr}$ sampling intervals [53].

This study showed that slight changes in formulation of the product can produce considerable differences in bioavailability of the drug and its eventual therapeutic effect. Therefore, the FDA recognized that tolbutamide products were prone to bioavailability problems and listed 12 manufactures' products as therapeutically equivalent to each other [53].

In another study, dissolution profile for 62 lots of tolbutamide tablets from 6 manufacturers showed that inter-lot and intra-lot variation was highly dependent on the interestingly, tablet response to aging at various relative humidity conditions exhibited large differences between manufactures. While the innovator's product showed no significant change on significant change on storage, the dissolution of some generic tablets decreased dramatically on aging under high humidity conditions. These studies indicated that if variations of such magnitude can occur between different formulations of the same drug, then the specifications regulating the bioavailability of these products needed reevaluation Also binding to albumin affects the positive outcome of tolbutamide [54].

There are many drugs for which bioequivalence have been suspected such as Papaverine, tetracycline hydrochloride and pentobarbital etc.

As mentioned earlier, bioequivalence has been shown to exist not only in products manufactured by different manufacturers, but in different batches of the same drug product from the same manufacturer. This can cause variations in the bioavailability of the drug and may cause potential therapeutic problems when a patient takes two bio-in equivalent drug products during the course of a therapy variations in the bioavailability can cause therapeutic failures.

\section{Conclusion}

Upon asking a question of whether bioavailability testing is necessary for all drug products? The answer is Yes, in principle. It is a very well-known fact that bioavailability studies are very time consuming and very expensive to conduct. Understandably, bioavailability testing for all products is economically difficult, and for some drugs such studies may not necessarily be essential. The question 
of determining bioavailability, however, is of particular interest for drug products which are included in one or more of the following categories where it is necessary to maintain a defined minimum effective concentration of drug in the (a) biological fluids during the course of therapy, (b) potent drugs; and (c) drugs which are poorly soluble in the gastrointestinal fluids (for the orally administrated drugs) or in the body fluids (for rugs which are administered rectally and by extravascular parenteral route).

Thus, candidates which are potential of reduced bioavailability are those drugs which exhibit the: following characteristics all drugs which have a low solubility at the site of administration or absorption (less than $0.3 . \%$ solubility) All drug products of which the active ingredient is too slowly or incompletely released, and (b). All drugs with narrow therapeutic index (c).

\section{Bibliography}

1. Caldwell J., et al. "An introduction to drug disposition: the basic principles of absorption, distribution, metabolism, and excretion". Toxicology and Pathology 23.2 (1995): 102-114.

2. Heyam Ali., et al. "Prevention of cap-locking of Syrup Product by Treating the Manufacturing Process with Citric Acid Monohydrate". International Journal of Pharmaceutical Chemistry 5.6 (2015): 218-226.

3. Takano R., et al. "Integrating drug permeability with dissolution profile to develop IVIVC". Biopharmaceutics and Drug Disposition 33.7 (2012): 354-365.

4. Mayersohn M. "Drug absorption". Journal of Clinical Pharmacology 27.9 (1987): 634-638.

5. Colomb Med (Cali)., et al. "In vitro-in vivo Pharmacokinetic correlation model for quality assurance of antiretroviral drugs". 46.3 (2015): 109-116.

6. Currie GM. "Pharmacology, Part 2: Introduction to Pharmacokinetics". Journal of Nuclear Medicine Technology 46.3 (2018): 221-230.

7. Lin L and Wong H. "Predicting Oral Drug Absorption: Mini Review on Physiologically-Based Pharmacokinetic Models". Pharmaceutics 9.4 (2017).
8. Esteban Walker and Amy S Nowacki. "Understanding Equivalence and Noninferiority Testing". Journal of General Internal Medicine 26.2 (2011): 192-196.

9. Barbara M Davit., et al. "BCS Biowaivers: Similarities and Differences among EMA, FDA, and WHO Requirements". AAPS Journal 18.3 (2016): 612-618.

10. Chen ML., et al. "The YLX, BCS BDDCS, and regulatory guidances". Pharmaceutical Research 28 (2011): 1774-1778.

11. G Levy. "Comparison of dissolution and absorption rates of different commercial aspirin tablets". Journal of Pharmaceutical Sciences 50 (2010): 388-392.

12. Heyam Ali., et al. "Extemporaneous Furosemide Suspensions for Pediatrics Use Prepared from Commercially Available Tablets". International Journal of Pharmacy and Pharmaceutical Research 5.2 (2016): 112-116.

13. M Havlik., et al. "Comparison of the pharmacokinetic profiles of soluble aspirin and solid paracetamol tablets in fed and fasted volunteers". Current Medical Research and Opinion 16 (2000): 115-124.

14. Stillings M., et al. "Comparison of the pharmacokinetic profiles of soluble aspirin and solid paracetamol tablets in fed and fasted volunteers". Current Medical Research and Opinion 16 (2000): 115-124.

15. Johnson BF and Bye C. "Maximal intestinal absorption of digoxin, and its relation to steady state plasma concentration". British Heart Journal 37.2 (1975): 203-208.

16. S D Ragendran., et al. "Comparison of target concentration intervention strategy with conventional dosing of digoxin". Indian Heart Journal 57.3 (2005): 265-267.

17. G Belier., et al. "Digitalis intoxication: A .4 prospective clinical study with serum level correlations". The New England Journal of Medicine 284 (1971): 989.

18. Johnson BF., et al. "Biological availability of digixon from Lanoxin produced in the United Kingdom". British Medical Journal 4.5888 (1973): 323-32a6. 
19. Greenwood H., et al. "A rapid, simple assay for digoxin". Journal of Clinical Pathology 27.6 (1974): 490-494.

20. R P Hayward., et al. "Comparison of digoxin and medigoxin in normal subjects". British Journal of Clinical Pharmacology 6.1 (1978): 81-86.

21. Lindenbaum J., et al. "Variation in biologic availability of digoxin from four preparations". The New England Journal of Medicine 285.24 (1971): 1344-1347.

22. Falch D., et al. "Comparative study of the absorption, plasma levels and urinary excretion of the "new" and the "old" Lanoxin". British Medical Journal 1.5855 (1973): 695-697.

23. Florence AT., et al. "The effect of particle size reduction on digoxin crystal properties". Journal of Pharmacy and Pharmacology 26.6 (1974): 479-480.

24. H Paul., et al. "Laboratory studies with nitrofurantoin: relationship between crystal size, urinary excretion in the rat and man, and emesis in dogs". Journal of Pharmaceutical Sciences 56 (1967): 882.

25. Hongixia Yu., et al. "Nitrofurantoin enteric pellets with high bioavailability based on aciform crystalline formation by wet milling". Pharmaceutical Development and Technology 20.4 (2015): 433-441.

26. Xin-Yi Teoh., et al. "Formulation strategy of nitrofurantoin: cocrystal or solid dispersion?". Pharmaceutical Development and Technology 25.2 (2020): 245-251.

27. Babiker M El-Haj., et al. "Linking Aromatic Hydroxy Metabolic Functionalization of Drug Molecules to Structure and Pharmacologic Activity”. Molecules 23.9 (2018): 2119.

28. Armen P Melikan., et al. "Bioavailability of 11 phenytoin products". Journal of Pharmacokinetics and Biopharmaceutics 5 (1977): 133-146.

29. "Generic substitution of antiepileptic drugs: a systemic review of prospective studies". Annals of Pharmacotherapy 45.11 (2011): 1406-1415.

30. D B Appleton., et al. "Blood phenytoin concentrations produced by ingestion of three different phenytoin preparations". Medical Journal of Australia (1972): 410.
31. Boréus LO., et al. "A comparison between microcrystalline and conventional phenytoin preperations: relative bioavailability and steady-state plasma concentrations". 223.4 (1980): 241249.

32. L Lund. "Clinical significance of generic inequivalence of three different pharmaceutical preparations of phenytoin". European Journal of Clinical Pharmacology 7 (1974): 119.

33. PJ Neuvonen. "Bioavailability of phenytoin: clinical pharmacokinetic and therapeutic implications". Clinical Pharmacokinetics 4.2 (1979): 91-103.

34. Julie Eve Desmarais., et al. "Switching from Brand-Name to Generic Psychotropic Medications: A Literature Review". CNS Neuroscience Therapy 6 (2011): 750-760.

35. Lin L and Wong H. "Predicting Oral Drug Absorption: Mini Review on Physiologically-Based Pharmacokinetic Models". Pharmaceutics 9.4 (2017).

36. Ang M., et al. "Evaluation of prednisolone acetate loaded subsequently implant for the treatment of recurrent uveitis in a rabbit model". Plos One 9.5 (2014).

37. G Levy., et al. "Studies on inactive prednisone tablets, USP XVI". American Journal of Health-System Pharmacy 21 (1964): 402.

38. Sullivan E., et al. "In vitro and in vivo availability of commercial prednisone tablets". Journal of Pharmaceutical Sciences 64 (1975): 1723.

39. K DiSanto and K DeSante. "Bioavailability and pharniacokinetics of prednisone in humans". Journal of Pharmaceutical Sciences 64 (1975): 109.

40. G Brice and H Hammer. "Therapeutic nonequivalence of oxytetracycline capsules". JAMA 19.208 (1969): 1189-1190.

41. D Blair., et al. "Biological availability of oxytetracycline hydrochloride capsules. A comparison of all manufacturing sources supplying the United States market". JAMA 251 (1971).

42. Larsen I., et al. "A randomised clinical trial on the efficacy of oxytetracycline dose through water medication of nursery pigs on diarrhoea, faecal shedding of Lawsonia intracellularis 
and average daily weight gain". Preventive Veterinary Medicine 123 (2016): 52-59.

43. H MacDonald., et al. "Physiological availability of various tetracyclines". 3 (1969) 76.

44. Græsbøll K., et al. "Effect of Tetracycline Dose and Treatment Mode on Selection of Resistant Coliform Bacteria in Nursery Pigs". Applied and Environmental Microbiology 83.12 (2017): e00538-517.

45. De Albuguergue Femandes SA. "Daily ingestion of tetracycline residues present in pasteurized milk. A public health problem". Environmental Science and Pollution Research 21.5 (2014): 3427-3434.

46. Kaare Græsbøll., et al. "Effect of Tetracycline Dose and Treatment Mode on Selection of Resistant Coliform Bacteria in Nursery Pigs". Applied and Environmental Microbiology 83.12 (2017): e00538-517.

47. B Catz., et al. "Clinically inactive Thyroid USP, A preliminary report”. The New England Journal of Medicine 226 (1962): 136.

48. A Ramos-Gabatin., et al. "In vivo comparison of levothyroxine preparations”. JAMA 247 (1982): 203.

49. Samrein Ahmed., et al. "Assessing the knowoledge of environmental risk factors for cancer among the UAE population: A pilot study". International Journal of Environmental Research and Public Health 17.9 (2020): 2984.

50. F Lu., et al. "A comparative study of some brands of tolbutamide in Canada. Part II. Pharmaceutical aspects". Canadian Medical Association Journal 92 (1965): 1166.

51. Olson SC and Ayres JW. "Effect of food and tablets age on relative bioavailability and pharmacodynamics of two tolbutamide products". Journal of Pharmaceutical Sciences 74.7 (1985): 735-740.

52. Ayres JW. "Lot- to- lot variation in dissolution of tolbutamide tablets". American Journal of Health-System Pharmacy 37.10 (1980): 1329-1332.

53. Szkudlarek Pentak D. "Effect of temperature on tolbutamide bind to glycated serum.32.albumin". Molecules 22.4 (2017): 569.
54. S Carminetsky. "Substitution for brand name drugs". Canadian Medical Association Journal 88 (1963): 950.

Volume 5 Issue 10 October 2021 (C) All rights are reserved by Heyam Saad Ali., et al. 